\title{
Pareto Optimal Control for Uncertain Markov Jump Linear Stochastic Systems
}

\author{
Hiroaki Mukaidani ${ }^{1}$, Ippo Ishibashi ${ }^{2}$, Shouhei Furuya ${ }^{3}$ \\ ${ }^{1}$ Graduate School of Engineering, Hiroshima University \\ 1-4-1 Kagamiyama, Higashi-Hiroshima, 739-8527 Japan \\ mukaida@hiroshima-u.ac.jp \\ ${ }^{2}$ Graduate School of Education, Hiroshima University \\ 1-1-1 Kagamiyama, Higashi-Hiroshima, 739-8524 Japan \\ ipishibashi@hiroshima-u.ac.jp \\ ${ }^{3}$ Hikarigaoka High School \\ 1-1 Hikarigaoka, Hikari, 743-0023 Japan \\ furuya.shouhei@ysn21.jp
}

\begin{abstract}
In this paper, a Pareto optimal strategy for uncertain Markovian linear stochastic system with multiple decision makers is investigated. By applying the guaranteed cost control principle, a set of conditions, wherein the stochastic system is exponentially mean-square stable (EMSS) and has a cost bound, is obtained using the stochastic algebraic Riccati inequality (SARI). In addition, the minimization problem of the cost bound is formulated. It is shown that the necessary conditions can be derived by a set of crosscoupled stochastic Riccati equations (CCSAREs).
\end{abstract}

Keywords: Pareto optimal control, uncertain Markovian jump linear stochastic systems, stochastic algebraic Riccati inequality (SARI), Karush-Kuhn-Tucker (KKT) conditions.

\section{Introduction}

Markov jump linear stochastic systems (MJLSSs) have been introduced to describe dynamics that are characterized by a stochastic process under random noise, unmodeled uncertainties, and abrupt changes of the operating points in various electrical and mechanical systems. In order to obtain a general representation of such sources of performance degradation, uncertain Markovian jump linear stochastic systems (UMJLSSs) have been extensively studied. Notably, UMJLSSs, unlike MJLSSs, can represent the deterministic uncertainties, which are independent of any stochastic process. Consequently, robust control techniques counteracting these system variations have been widely investigated in recent years, and a variety of different approaches to designing the controller has been adopted over the last decade. In [1], the $H_{\infty}$ problem for UMJLSS governed by Itô stochastic differential equations has been considered. Necessary and sufficient conditions for the existence of the infinite horizon mixed $\mathrm{H}_{2} / \mathrm{H}_{\infty}$ control of stochastic Markovian jumps have been obtained by means of a pair of coupled Riccati-type equations [2].

With the increasing demand for control strategies to be applied, for instance, to multi-agent systems, dynamic games for a class of Markov jump stochastic systems have received considerable attention in recent years. The Nash equilibrium strategy set for multiparameter singularly perturbed Markov jump stochastic systems has been established by solving the cross-coupled stochastic algebraic Riccati equations (CSAREs) [3]. $H_{2} / H_{\infty}$ control problem for a class of Markov jump linear stochastic systems with $(x, u, v)$-dependent noise involving multiple decision makers has been addressed [4]. Nash games and the related $H_{2} / H_{\infty}$ control for a class of linear stochastic systems with Markovian jump parameters, both in finite-time and infinite-time horizon, have been studied [5]. In [6], the stochastic non-cooperative differential game theory of generalized linear Markov jump systems and its applications have been studied extensively. Even though fruitful results have been obtained in these studies, there is no discussion on robust dynamic games taking into account unmodeled deterministic uncertainties and external disturbances. The reason is that the strategy set in dynamic games is not unique for describing a system's unmodeled dynamics and the exact cost cannot be computed due to deterministic uncertainties. 
In this paper, the Pareto optimal strategy for UMJLSS involving multiple decision makers is discussed. In particular, a novel robust strategy set against Markov switching models and deterministic uncertainty is formulated. By applying the well-known guaranteed cost control principle [7], the conditions, wherein the stochastic system is exponentially meansquare stable (EMSS) and has a cost bound, are given by the stochastic algebraic Riccati inequality (SARI). In contrast to the existing control strategy $[3,4,5,6]$, it is more difficult for dynamic games to attain their equilibrium due to the complexity of the calculation of their cost bound. Hence, this research is a non-trivial extension of the existing results. Our results can be summarized as follows. Initially, a preliminary result about single decision makers is obtained. Namely, the SARI is established. This implies that the closed-loop UMJLSS are exponentially mean-square stable and their performance has an upper cost bound. The minimization problem of the cost bound is formulated and the necessary conditions are derived from the set of cross-coupled stochastic Riccati equations (CCSAREs) by means of the KarushKuhn-Tucker (KKT) conditions.

Notation: The notations used in this paper are fairly standard. $\mathbb{E}\left[\cdot \mid r_{t}=i\right]$ stands for the conditional expectation operator with respect to the event $\left\{r_{t}=i\right\} \cdot \chi_{A}$ denotes indicator function. $\mathbb{M}_{n, m}^{S}$ denotes space of all $\mathbf{S}=(S(1), \ldots, S(s))$ with $S(i)$ being $n \times m$ matrix, $i \in \mathcal{D}, \mathcal{D}=\{1,2, \ldots, s\}$. Moreover, the component of $\mathbf{S}+\mathbf{T U}$ is defined as $\mathbf{S}+\mathbf{T U}=$ $(S(1)+T(1) U(1), \ldots, S(s)+T(s) U(s))$.

\section{Definition and Preliminaries}

Let $\left(\Omega, \mathcal{F},\left\{\mathcal{F}_{t}\right\}_{t \geq 0}, \mathbf{P}\right)$ be a given filtered probability space where there exists a standard one-dimensional Wiener process $w(t), t \geq 0$, and a right continuous homogeneous Markov process $r_{t}, t \geq 0$ with state space $\mathcal{D}=\{1,2, \ldots, s\}$. It is assumed that $\{w(t)\}_{t \geq 0}$ and $\left\{r_{t}\right\}_{t \geq 0}$ are independent stochastic processes. Moreover, it is assumed that the Markov process $r_{t}$ has the transition probabilities given by

$$
\boldsymbol{P}\left\{r_{t+h}=j \mid r_{t}=i\right\}=\left\{\begin{aligned}
\pi_{i j} h+o(h), & \text { if } i \neq j \\
1+\pi_{i i} h+o(h), & \text { if else }
\end{aligned}\right.
$$

where $\lim _{h \rightarrow 0} o(h) / h=0, \pi_{i j} \geq 0$ for $i \neq j$ and $\pi_{i i}=-\sum_{j=1, j \neq i}^{S} \pi_{i j}[8]$.

Definition 1. [1] We consider the following linear stochastically controlled uncertain system with Markovian jumps

$$
d x(t)=\left[A\left(r_{t}, t\right) x(t)+B\left(r_{t}, t\right) u(t)\right] d t+A_{p}\left(r_{t}\right) x(t) d w(t)
$$

where $x(t) \in \mathbb{R}^{n}$ represents the state vector, $u(t) \in \mathbb{R}^{m}$ represents the control inputs and $A\left(r_{t}, t\right), A_{p}\left(r_{t}\right) \in \mathbb{R}^{n \times n}$ and $B\left(r_{t}, t\right) \in \mathbb{R}^{n \times m}$ are matrices with the following forms:

$$
\begin{aligned}
& A\left(r_{t}, t\right)=A\left(r_{t}\right)+D\left(r_{t}\right) F\left(r_{t}, t\right) E_{a}\left(r_{t}\right), \\
& B\left(r_{t}, t\right)=B\left(r_{t}\right)+D\left(r_{t}\right) F\left(r_{t}, t\right) E_{b}\left(r_{t}\right),
\end{aligned}
$$

with

$$
F^{T}\left(r_{t}, t\right) F\left(r_{t}, t\right) \leq I_{n_{a}}
$$

The coefficient matrices $\mathbf{A}, \mathbf{A}_{p} \in \mathbb{M}_{n, n}^{s}$ and $\mathbf{B} \in \mathbb{M}_{n, m}^{s}$ are defined by $A(i), B(i), A_{p}(i), D(i), E_{a}(i)$ and $E_{b}(i), i \in \mathcal{D}$ with compatible dimensions. $F\left(r_{t}, t\right) \in \mathbb{R}^{n_{p} \times n_{a}}$ are uncertainty matrices.

The uncertain stochastic system (2) is called stochastic stabilizable in the mean-square sense. If there exists a feedback control 


$$
u(t)=\sum_{i=1}^{s} K(i) x(t) \chi_{r_{t}=i}
$$

such that for any initial state $x(0)=x_{0}, r_{0}=i$, the closed-loop system

$$
d x(t)=\left[A\left(r_{t}, t\right) x(t)+B\left(r_{t}, t\right) K\left(r_{t}\right)\right] d t+A_{p}\left(r_{t}\right) x(t) d w(t)
$$

is exponentially mean-square stable (EMSS), i.e.

$$
\mathbb{E}\left[\|x(t)\|^{2}\right] \leq \beta e^{-\alpha\left(t-t_{0}\right)} \mathbb{E}\left[\left\|x\left(t_{0}\right)\right\|^{2}\right], \exists \alpha, \beta>0
$$

The following matrix inequality is well-known.

Lemma 1. [9] Let $D \in \mathbb{R}^{n \times p}, E \in \mathbb{R}^{q \times n}$ and $F \in \mathbb{R}^{p \times q}$ be matrices satisfying $F^{T} F \leq I_{q}$. For any $\mu>0$, the following inequality holds.

$$
E^{T} F^{T} D^{T}+D F E \leq \mu D D^{T}+\mu^{-1} E^{T} E .
$$

The following result is used to derive the preliminary contribution.

Theorem 1. Consider the following autonomous UMJLSS:

$$
\begin{aligned}
d x(t) & =A\left(r_{t}, t\right) x(t) d t+A_{p}\left(r_{t}\right) x(t) d w(t) \\
& =\left[A\left(r_{t}\right)+D\left(r_{t}\right) F\left(r_{t}, t\right) E_{a}\left(r_{t}\right)\right] x(t) d t+A_{p}\left(r_{t}\right) x(t) d w(t), x(0)=x_{0}
\end{aligned}
$$

and the cost function.

$$
\bar{J}\left(x(0), r_{0}\right)=\mathbb{E}\left[\int_{0}^{\infty} x^{T}(t) Q\left(r_{t}\right) x(t) d t \mid r_{0}=i\right]
$$

where $Q\left(r_{t}\right)=Q^{T}\left(r_{t}\right)>0$.

We assume that there exist positive definite real symmetric matrices $P(i)=P^{T}(i)>0$ and positive scalar parameters $\mu_{i}>0, i=1, \ldots, s$ such that the matrix inequalities (11) hold.

$$
\widetilde{\Xi}_{i}\left(\mathbf{P}, \mu_{i}\right) \leq 0, i=1, \ldots, s,
$$

where

$$
\begin{aligned}
\widetilde{\Xi}_{i}\left(\mathbf{P}, \mu_{i}\right):= & P(i) A(i)+A^{T}(i) P(i)+\mu_{i} P(i) D(i) D^{T}(i) P(i)+\mu_{i}^{-1} E_{a}^{T}(i) E_{a}(i) \\
& +\sum_{j=1}^{s} \pi_{i j} P(j)+A_{p}^{T}(i) P(i) A_{p}(i)+Q(i) .
\end{aligned}
$$

Then, we have

(i) The UMJLSS (9) is EMSS.

(ii) The cost function has the following upper bound. 


$$
\mathbb{E}\left[\int_{0}^{\infty} x^{T}(t) Q\left(r_{t}\right) x(t) d t \mid r_{0}=i\right] \leq \mathbb{E}\left[x^{T}(0) P\left(r_{0}\right) x(0) \mid r_{0}=i\right]
$$

Proof: We first define the following Lyapunov function candidate with $P(i)=P^{T}(i)>0$.

$$
V(x, i, t):=x^{T}(t) P(i) x(t) .
$$

Using Lemmas 1 and 2 for the UMJLSS, (9) implies the following equation.

$$
\begin{aligned}
& \mathcal{D} V(x, i, t)+x^{T}(t) Q(i) x(t) \\
& =x^{T}(t)\left(P(i)\left[A(i)+D(i) F(i, t) E_{a}(i)\right]+\left[A(i)+D(i) F(i, t) E_{a}(i)\right]^{T} P(i)\right) x(t) \\
& \quad+\sum_{j=1}^{s} \pi_{i j} x^{T}(t) P(j) x(t)+x^{T}(t) A_{p}^{T}(i) P(i) A_{p}(i) x(t)+x^{T}(t) Q(i) x(t) \\
& \leq x^{T}(t) \widetilde{\Xi}_{i}\left(\mathbf{P}, \mu_{i}\right) x(t) .
\end{aligned}
$$

Hence, if $\widetilde{\Xi}_{i}\left(\mathbf{P}, \mu_{i}\right) \leq 0$ holds, then, since $Q(i)>0$, the uncertain autonomous stochastic system is EMSS. Therefore, we have $\mathbb{E}\left[V(x(\infty), i, \infty) \mid r_{0}=i\right]=0$. Thus, integrating both sides of the above equation and using $\mathbb{E}\left[V(x(\infty), i, \infty) \mid r_{0}=\right.$ i] $=0$, we obtain

$$
\bar{J}\left(x(0), r_{0}\right)-\mathbb{E}\left[V\left(x(0), r_{0}, 0\right) \mid r_{0}=i\right] \leq 0 .
$$

Therefore, if (13) holds, then the proof of Theorem 1 is complete.

\subsection{Necessary Conditions Obtained Using KKT Conditions}

Without loss of generality, we may assume that the initial condition satisfies the following equation.

$$
\mathbb{E}\left[x(0) x^{T}(0)\right]=I_{n}
$$

We introduce the main contribution of this paper.

Theorem 2. We consider the UMJLSS and the cost function (18).

$$
\begin{aligned}
& \left.d x(t)=\left(\left[A\left(r_{t}\right)+D\left(r_{t}\right) F\left(r_{t}, t\right) E_{a}\left(r_{t}\right)\right] x(t)+\left[B\left(r_{t}\right) u(t)+D\left(r_{t}\right) F\left(r_{t}, t\right) E_{b}\left(r_{t}\right)\right] u(t)\right]\right) x(t) d t \\
& +A_{p}\left(r_{t}\right) x(t) d w(t) \\
& u(t)=\sum_{i=1}^{s} K(i) x(t) \chi_{r_{t}=i}, \\
& J\left(x(0), r_{0}\right)=\mathbb{E}\left[\int_{0}^{\infty} x^{T}(t)\left[Q\left(r_{t}\right)+K^{T}\left(r_{t}\right) R\left(r_{t}\right) K\left(r_{t}\right)\right] x(t) d t \mid r_{0}=i\right] \text {, }
\end{aligned}
$$

where $Q\left(r_{t}\right)=Q^{T}\left(r_{t}\right) \geq 0$ and $R\left(r_{t}\right)=R^{T}\left(r_{t}\right)>0$.

We assume that there exist positive definite real symmetric matrices $X(i)=X^{T}(i)>0$ and positive scalar parameters $\mu_{i}>0, i=1, \ldots, s$ such that

$$
\Xi_{i}\left(\mathbf{X}, K(i), \mu_{i}\right) \leq 0, i=1, \ldots, s,
$$


where

$$
\begin{aligned}
\Xi_{i}\left(\mathbf{X}, K(i), \mu_{i}\right):= & X(i)[A(i)+B(i) K(i)]+[A(i)+B(i) K(i)]^{T} X(i)+\mu_{i} X(i) D(i) D^{T}(i) X(i) \\
& +\mu_{i}^{-1}\left[E_{a}(i)+E_{b}(i) K(i)\right]^{T}\left[E_{a}(i)+E_{b}(i) K(i)\right]+\sum_{j=1}^{s} \pi_{i j} X(j)+A_{p}^{T}(i) X(i) A_{p}(i) \\
& +Q(i)+K^{T}(i) R(i) K(i) .
\end{aligned}
$$

Then, we have

$$
J\left(x(0), r_{0}\right) \leq \mathbb{E}\left[x^{T}(0) X\left(r_{0}\right) x(0) \mid r_{0}=i\right]=\operatorname{Tr}\left[X\left(r_{0}\right)\right]
$$

Let us now consider the minimization problem of the cost bound of (20). If the following conditions hold

i. $\quad\left[A(i)+B(i) K(i), A_{p}(i) \mid I_{n}\right]$ is stochastically detectable.

ii. $\quad\left(A(i)+B(i) K(i), A_{p}(i)\right)$ is stable.

and $v_{i}^{*}$ is a local minimum that satisfies the constraint qualification, then there exist unique positive definite solutions $\mathbf{G}^{*}>0, \mathbf{X}^{*}>0, \mathbf{K}^{*}$ and $\mu_{i}^{*}$ such that the following cross-coupled stochastic algebraic Riccati equations (CSAREs) are satisfied.

where

$$
\begin{aligned}
& \Xi_{i}\left(\mathbf{X}^{*}, K^{*}(i), \mu_{i}^{*}\right)=0, \\
& \Gamma_{i}\left(G^{*}(i), X^{*}(i), K^{*}(i), \mu_{i}^{*}\right)=0, \\
& \Phi_{i}\left(G^{*}(i), X^{*}(i), K^{*}(i), \mu_{i}^{*}\right)=0, \\
& v_{i}^{*}=\left(\left[\operatorname{vec} G^{*}(i)\right]^{T},\left[\operatorname{vec} X^{*}(i)\right]^{T},\left[\operatorname{vec} K^{*}(i)\right]^{T}, \mu_{i}^{*}\right)^{T},
\end{aligned}
$$

$\Gamma_{i}\left(G(i), X(i), K(i), \mu_{i}\right)$

$$
\begin{aligned}
:= & {[A(i)+B(i) K(i)] G(i)+G(i)[A(i)+B(i) K(i)]^{T}+\mu_{i}\left[D(i) D^{T}(i) X(i) G(i)+G(i) X(i) D(i) D^{T}(i)\right] } \\
& +\sum_{j=1}^{s} \pi_{j i} G(j)+A_{p}(i) G(i) A_{p}^{T}(i)+I_{n}
\end{aligned}
$$

$\Phi_{i}\left(G(i), X(i), K(i), \mu_{i}\right)$

$$
:=\operatorname{Tr}\left[D^{T}(i) X(i) G(i) X(i) D(i)\right]-\mu_{i}^{-2} \operatorname{Tr}\left[\left[E_{a}(i)+E_{b}(i) K(i)\right] G(i)\left[E_{a}(i)+E_{b}(i) K(i)\right]^{T}\right] .
$$

In other words, let $v_{i}^{*}$ be the solution set that gives a local minimum. Then the controls are given by

$$
u(t)=u_{\mathrm{opt}}(t)=\sum_{i=1}^{s} K^{*}(i) x(t) \chi_{r_{t}=i}
$$

where

$$
K^{*}(i)=-\left[R(i)+\mu_{i}^{*} E_{b}^{T}(i) E_{b}(i)\right]^{-1}\left[B^{T}(i) X(i)+\mu_{i}^{*} E_{b}^{T}(i) E_{a}(i)\right]
$$

Furthermore, the minimization of the cost bound under the feedback controls (22) is attained, and the CSAREs (21) hold. That is,

$$
\min _{X\left(r_{0}\right)} \operatorname{Tr}\left[X\left(r_{0}\right)\right]=\operatorname{Tr}\left[X^{*}\left(r_{0}\right)\right]
$$


Proof: The first part of Theorem 2 can be proved using techniques similar to those of Theorem 1. Therefore, this part will be omitted. The rest of the proof can be completed using the Karush-Kuhn-Tucker condition [11]. The minimization problem of the cost bound (20) subject to constraint (19) can be modified as follows. Let us consider the Lagrangian $L$.

$$
L=L\left(\mathbf{G}, \mathbf{X}, \mathbf{K}, \mu_{i}\right)=\operatorname{Tr}[X(i)]+\sum_{j=1}^{s} \operatorname{Tr}\left[G(j) \Xi_{j}\left(\mathbf{X}, K(j), \mu_{j}\right)\right]
$$

where $r_{0}=i, G(i)$ is the symmetric matrix of Lagrange multipliers.

It is clear that $\operatorname{Tr}[X(i)]$ and $\Xi_{i}\left(\mathbf{X}, K(j), \mu_{j}\right)$ are continuously differentiable at the point $v_{i}^{*}$. Using the KKT conditions, we have

$$
\begin{aligned}
& \Xi_{i}\left(\mathbf{X}, K(i), \mu_{i}\right) \leq 0, G(i) \geq 0, G(i) \Xi_{i}\left(\mathbf{X}, K(i), \mu_{i}\right)=0, \\
& \frac{\partial L}{\partial X(i)}=\Gamma_{i}\left(G(i), X(i), K(i), \mu_{i}\right)=0, \\
& \frac{\partial L}{\partial K(i)}=2\left(B^{T}(i) X(i)+\mu_{i} E_{b}^{T}(i) E_{a}(i)+\left[R(i)+\mu_{i} E_{b}^{T}(i) E_{b}(i)\right] K(i)\right) G(i)=0, \\
& \frac{\partial L}{\partial \mu_{i}}=\Phi_{i}\left(G(i), X(i), K(i), \mu_{i}\right) .
\end{aligned}
$$

Applying conditions $\mathrm{i}$ and ii to the generalized cross-coupled stochastic Sylvester equations (GCCSSEs) (25b), it immediately follows that they have a unique positive definite solution $G^{*}(i)>0$. Hence, from equation $(25 \mathrm{c})$, we can derive the controls (22). From equation (25a), we know that $\Xi_{i}\left(\mathbf{X}^{*}, K^{*}(i), \mu_{i}^{*}\right)=0$ since $G^{*}(i)>0$. From the remaining equation, (25d) holds. This completes the proof of Theorem 2.

In the next section, we study the Pareto optimal control problem for a class of UMJLSSs with multiple decision makers.

\section{Pareto Optimal Control Strategy}

We consider the following UMJLSS with $N$-decision makers and the cost functions.

$$
\begin{aligned}
& d x(t)=\left.\left(\left[A\left(r_{t}\right)+D\left(r_{t}\right) F\left(r_{t}, t\right) E_{a}\left(r_{t}\right)\right] x(t)+\sum_{k=1}^{N}\left[B_{k}\left(r_{t}\right) u(t)+D\left(r_{t}\right) F\left(r_{t}, t\right) E_{b k}\left(r_{t}\right)\right] u_{k}(t)\right]\right) \\
& \times x(t) d t+A_{p}\left(r_{t}\right) x(t) d w(t), \\
& J_{k}\left(x(0), r_{0}\right)=\mathbb{E}\left[\int_{0}^{\infty} x^{T}(t)\left[Q_{k}\left(r_{t}\right)+K_{k}^{T}\left(r_{t}\right) R_{k}\left(r_{t}\right) K_{k}\left(r_{t}\right)\right] x(t) d t \mid r_{0}=i\right],
\end{aligned}
$$

where $Q_{k}\left(r_{t}\right)=Q_{k}^{T}\left(r_{t}\right) \geq 0$ and $R_{k}\left(r_{t}\right)=R_{k}^{T}\left(r_{t}\right)>0$. Moreover, the coefficients $\mathbf{B}_{k} \in \mathbb{M}_{n, m}^{s}$ with $B_{k}(i)$ and $E_{b k}(i)$, $i \in \mathcal{D}$ being constant matrices of compatible dimensions. A Pareto solution is a set $\left(\mathbf{u}_{1}, \ldots, \mathbf{u}_{N}\right)$, which minimizes

$$
J\left(\mathbf{u}_{1}, \ldots, \mathbf{u}_{N}, x(0), r_{0}\right)=\sum_{k=1}^{N} \gamma_{k} J_{k}\left(x(0), r_{0}\right), 0<\gamma_{k}<1, \sum_{k=1}^{N} \gamma_{k}=1
$$

for some $\gamma_{k}, k=1, \ldots, N[12]$. Without loss of generality, the strategies are restricted as follows: 


$$
u_{k}(t)=\sum_{i=1}^{s} K_{k}(i) x(t) \chi_{r_{t}=i}, k=1, \ldots, N
$$

The definition of the Pareto optimal control for UMJLSS with multiple decision makers is given below.

Definition 2. A linear feedback strategy expressed as (28) is said to be the Pareto optimal control with cost function (27) if the closed-loop system is EMSS, and the closed-loop value of (27) satisfies the bound

$$
J\left(\mathbf{u}_{1}, \ldots, \mathbf{u}_{N}, x(0), r_{0}\right) \leq \mathbf{J}
$$

for all admissible uncertainties.

The aim of this paper is to introduce the Pareto optimal control strategies (28) for UMJLSS (26a) with multiple decision makers.

\subsection{Main Result}

Theorem 3. We consider the UMJLSS (26a) and the cost function (26b). We assume that there exist positive definite real symmetric matrices $X(i)=X^{T}(i)>0$ and positive scalar parameters $\mu_{i}>0, i=1, \ldots, s$ such that

$$
\Lambda_{i}\left(\mathbf{X}, K_{1}(i), \ldots, K_{N}(i), \mu_{i}\right) \leq 0, i=1, \ldots, s
$$

where

$$
\begin{aligned}
& \Lambda_{i}\left(\mathbf{X}, K_{1}(i), \ldots, K_{N}(i), \mu_{i}\right) \\
& :=X(i)\left[A(i)+\sum_{k=1}^{N} B_{k}(i) K_{k}(i)\right]+\left[A(i)+\sum_{k=1}^{N} B_{k}(i) K_{k}(i)\right]^{T} X(i)+\mu_{i} X(i) D(i) D^{T}(i) X(i) \\
& \quad+\mu_{i}^{-1}\left[E_{a}(i)+\sum_{k=1}^{N} E_{b k}(i) K_{k}(i)\right]^{T}\left[E_{a}(i)+\sum_{k=1}^{N} E_{b k}(i) K_{k}(i)\right]+\sum_{j=1}^{s} \pi_{i j} X(j)+A_{p}^{T}(i) X(i) A_{p}(i) \\
& \quad+\sum_{k=1}^{N} \gamma_{k}\left[Q_{k}(i)+K_{k}^{T}(i) R_{k}(i) K_{k}(i)\right] .
\end{aligned}
$$

Then, we have

$$
J\left(\mathbf{u}_{1}, \ldots, \mathbf{u}_{N}, x(0), r_{0}\right) \leq \mathbf{J}=\mathbb{E}\left[x^{T}(0) X\left(r_{0}\right) x(0) \mid r_{0}=i\right]=\operatorname{Tr}\left[X\left(r_{0}\right)\right]
$$

Let us now consider the minimization problem of the cost bound of (31). If the following conditions hold

i. $\left[A(i)+\sum_{k=1}^{N} B_{k}(i) K_{k}(i), A_{p}(i) \mid I_{n}\right]$ is stochastically detectable.

ii. $\left(A(i)+\sum_{k=1}^{N} B_{k}(i) K_{k}(i), A_{p}(i)\right)$ is stable.

and $v_{i}^{*}$ is a local minimum that satisfies the constraint qualification, then there exist unique positive definite solutions $\mathbf{H}^{*}>0, \mathbf{X}^{*}>0, \mathbf{K}_{k}^{*}$ and $\mu_{i}^{*}$ such that the following CSAREs (32) are satisfied.

$$
\begin{aligned}
& \Lambda_{i}\left(\mathbf{X}^{*}, K_{1}^{*}(i), \ldots, K_{N}^{*}(i), \mu_{i}^{*}\right)=0, \\
& \Delta_{i}\left(\mathbf{H}^{*}, X^{*}(i), K_{1}^{*}(i), \ldots, K_{N}^{*}(i), \mu_{i}^{*}\right)=0,
\end{aligned}
$$


here

$$
\begin{aligned}
& \Psi_{i}\left(H^{*}(i), X^{*}(i), K_{1}^{*}(i), \ldots, K_{N}^{*}(i), \mu_{i}^{*}\right)=0, \\
& v_{i}^{*}=\left(\left[\operatorname{vec} G^{*}(i)\right]^{T},\left[\operatorname{vec} X^{*}(i)\right]^{T},\left[\operatorname{vec} K_{1}^{*}(i)\right]^{T}, \ldots,\left[\operatorname{vec} K_{N}^{*}(i)\right]^{T}, \mu_{i}^{*}\right)^{T},
\end{aligned}
$$

$$
\begin{aligned}
& \Delta_{i}\left(\mathbf{H}, X(i), K_{1}(i), \ldots, K_{N}(i), \mu_{i}\right) \\
& :=\left[A(i)+\sum_{k=1}^{N} B_{k}(i) K_{k}(i)\right] H(i)+H(i)\left[A(i)+\sum_{k=1}^{N} B_{k}(i) K_{k}(i)\right]^{T} \\
& \quad+\mu_{i}\left[D(i) D^{T}(i) X(i) H(i)+H(i) X(i) D(i) D^{T}(i)\right]+\sum_{j=1}^{s} \pi_{j i} H(j)+A_{p}(i) H(i) A_{p}^{T}(i)+I_{n},
\end{aligned}
$$

$$
\begin{aligned}
& \Psi_{i}\left(H(i), X(i), K_{1}(i), \ldots, K_{N}(i), \mu_{i}\right) \\
& :=\operatorname{Tr}\left[D^{T}(i) X(i) H(i) X(i) D(i)\right]-\mu_{i}^{-2} \operatorname{Tr}\left[\left[E_{a}(i)+\sum_{k=1}^{N} E_{b k}(i) K_{k}(i)\right] H(i)\left[E_{a}(i)+\sum_{k=1}^{N} E_{b k}(i) K_{k}(i)\right]^{T}\right] .
\end{aligned}
$$

Namely, if $v_{i}^{*}$ is the solution set that yields a local minimum, then the controls are given

$$
u_{k}(t)=u_{k o p t}(t)=\sum_{i=1}^{s} K_{k}^{*}(i) x(t) \chi_{r_{t}=i}
$$

where

$$
B_{k}^{T}(i) X^{*}(i)+\mu_{i}^{*} E_{b k}^{T}(i) E_{a}(i)+\gamma_{i} R(i) K_{k}^{*}(i)+\mu_{i}^{*} \sum_{k=1}^{N} E_{b k}^{T}(i) E_{b k}(i) K_{k}^{*}(i)=0 .
$$

Further, the minimization of the cost bound under the feedback controls (33) is attained, and (32) holds. That is,

$$
\min _{X\left(r_{0}\right)} \operatorname{Tr}\left[X\left(r_{0}\right)\right]=\operatorname{Tr}\left[X^{*}\left(r_{0}\right)\right]
$$

Proof: The proof of Theorem 3 can be completed using a technique similar to that in Theorem 2. Let us consider the Lagrangian $M$.

$$
M=M\left(\mathbf{H}, \mathbf{X}, \mathbf{K}_{1}, \ldots, \mathbf{K}_{N}, \mu_{i}\right)=\operatorname{Tr}[X(i)]+\sum_{j=1}^{s} \operatorname{Tr}\left[H(j) \Lambda_{j}\left(\mathbf{X}, K_{1}(j), \ldots, K_{N}(j), \mu_{j}\right)\right],
$$

where $r_{0}=i, H(i)$ is the symmetric matrix of Lagrange multipliers.

Using the KKT conditions, we have

$$
\begin{aligned}
& \Lambda_{i}\left(\mathbf{X}, K_{1}(i), \ldots, K_{N}(i), \mu_{i}\right) \leq 0, H(i) \geq 0, H(i) \Lambda_{i}\left(\mathbf{X}, K_{1}(i), \ldots, K_{N}(i), \mu_{i}\right)=0, \\
& \frac{\partial M}{\partial X(i)}=\Delta_{i}\left(H(i), K_{1}(i), \ldots, K_{N}(i), \mu_{i}\right)=0, \\
& \frac{\partial M}{\partial K_{k}(i)}=2\left(B_{k}^{T}(i) X^{*}(i)+\mu_{i} E_{b k}^{T}(i) E_{a}(i)+\gamma_{i} R(i) K_{k}(i)+\mu_{i} \sum_{k=1}^{N} E_{b k}^{T}(i) E_{b k}(i) K_{k}(i)\right) H(i)=0, \\
& \frac{\partial M}{\partial \mu_{i}}=\Psi_{i}\left(H(i), X(i), K_{k}(i), \ldots, K_{N}(i), \mu_{i}\right) .
\end{aligned}
$$


By using arguments as in the proof of Theorem 2, we can prove Theorem 3.

\section{Numerical Example}

Considering the following coefficient matrices in the UMJLSS (26), where it is assumed that the UMJLSS has three decision makers.

$$
\begin{aligned}
A(1) & =\left[\begin{array}{cc}
-2 & 1 \\
0 & -2
\end{array}\right], A_{p}(1)=\left[\begin{array}{cc}
0.24 & 0 \\
-0.87 & 0.2
\end{array}\right], B_{1}(1)=\left[\begin{array}{c}
1 \\
1
\end{array}\right], B_{2}(1)=\left[\begin{array}{c}
1 \\
0.5
\end{array}\right], B_{3}(1)=\left[\begin{array}{l}
0 \\
1
\end{array}\right], \\
A(2) & =\left[\begin{array}{cc}
-2 & 1 \\
1 & -1
\end{array}\right], A_{p}(2)=\left[\begin{array}{cc}
-0.24 & 0 \\
1 & 0.2
\end{array}\right], B_{1}(2)=\left[\begin{array}{c}
0.5 \\
1
\end{array}\right], B_{2}(2)=\left[\begin{array}{c}
1 \\
-0.5
\end{array}\right], B_{3}(2)=\left[\begin{array}{c}
0.5 \\
1
\end{array}\right], \\
D(1) & =\left[\begin{array}{c}
0 \\
0.1
\end{array}\right], D(2)=\left[\begin{array}{c}
0 \\
0.2
\end{array}\right], E_{a}(1)=E_{a}(2)=\left[\begin{array}{ll}
0.01 & 0.01
\end{array}\right], \\
E_{b 1}(1) & =E_{b 1}(2)=0.01, E_{b 2}(1)=E_{b 2}(2)=0.02, E_{b 3}(1)=E_{b 3}(2)=0.03, \\
x(0) & =\left[\begin{array}{cc}
1 & -0.5
\end{array}\right]^{T}, \gamma_{1}=0.5, \gamma_{2}=\gamma_{3}=0.25, \\
Q_{1}(1) & =Q_{1}(2)=I_{2}, Q_{2}(1)=Q_{2}(2)=2 I_{2}, Q_{3}(1)=Q_{3}(2)=3 I_{2}, \\
R_{1}(1) & =R_{1}(2)=R_{2}(1)=R_{2}(2)=R_{3}(1)=R_{3}(2)=1 .
\end{aligned}
$$

By using the recursion given using the design procedure, the set of solutions are obtained

$$
\begin{aligned}
P(1) & =\left[\begin{array}{ll}
3.8289 \mathrm{e}-1 & 8.3465 \mathrm{e}-2 \\
8.3465 \mathrm{e}-2 & 4.1783 \mathrm{e}-1
\end{array}\right], P(2)=\left[\begin{array}{ll}
4.0930 \mathrm{e}-1 & 1.4887 \mathrm{e}-1 \\
1.4887 \mathrm{e}-1 & 4.6348 \mathrm{e}-1
\end{array}\right], \\
S(1) & =\left[\begin{array}{ll}
1.9490 \mathrm{e}-1 & 4.8546 \mathrm{e}-2 \\
4.8546 \mathrm{e}-2 & 2.4436 \mathrm{e}-1
\end{array}\right], S(2)=\left[\begin{array}{ll}
2.0542 \mathrm{e}-1 & 2.3014 \mathrm{e}-2 \\
2.3014 \mathrm{e}-2 & 1.4977 \mathrm{e}-1
\end{array}\right], \\
K_{1}(1) & =\left[\begin{array}{lll}
-2.7920 \mathrm{e}-1 & -2.6833 \mathrm{e}-1
\end{array}\right], K_{1}(2)=\left[\begin{array}{ll}
5.2598 \mathrm{e}-2 & -7.2742 \mathrm{e}-1
\end{array}\right], \\
K_{2}(1) & =\left[\begin{array}{lll}
-9.3558 \mathrm{e}-1 & -1.5774 \mathrm{e}-2
\end{array}\right], K_{2}(2)=\left[\begin{array}{ll}
-6.2710 \mathrm{e}-1 & 8.4923 \mathrm{e}-1
\end{array}\right], \\
K_{3}(1) & =\left[\begin{array}{lll}
9.6929 \mathrm{e}-1 & -2.0301 \mathrm{e}-1
\end{array}\right], K_{3}(2)=\left[\begin{array}{ll}
-8.5428 \mathrm{e}-1 & -1.1314
\end{array}\right], \\
\mu_{1} & =3.6153 \mathrm{e}-1, \mu_{2}=3.5877 \mathrm{e}-1 .
\end{aligned}
$$

\section{Conclusion}

In this study, the Pareto optimal control problem for UMJLSS with multiple decision makers was investigated. Necessary conditions for the existence of a Pareto strategy set that consist of CSAREs were derived by means of KKT conditions. Consequently, it was shown that the Pareto optimal strategy set can be constructed by solving the CSAREs.

\section{References}

[1] E. K. Boukas, Stochastic Switching Systems: Analysis and Design. Boston, MA: Birkhauser, 2006.

[2] Y. Huanga, W. Zhang and G. Feng, "Infinite horizon $H_{2} / H_{\infty}$ control for stochastic systems with Markovian jumps," Automatica, vol. 44, no.3, pp. 857-863, 2008.

[3] H. Mukaidani and T. Yamamoto, "Nash strategy for multiparameter singularly perturbed Markov jump stochastic systems," IET Control Theory \& Applications, vol. 6, no. 14, pp. 2337-2345, 2012.

[4] H. Mukaidani, H. Xu, T. Yamamoto and V. Dragan, "Static output feedback $H_{2} / H_{\infty}$ control of infinite horizon Markov jump linear stochastic systems with multiple decision makers," in Proceedings of the IEEE International Conference on Decision and Control, Maui, Hawaii, 2012, pp. 6003-6008.

[5] H.-N. Zhu, C.-K. Zhang and N. Bin, "Stochastic Nash games for Markov jump linear systems with state- and control-dependent noise," J. the Operations Research Society of China, vol. 2, no. 4, pp. 481-498, 2014.

[6] C.-K. Zhang, H.-N. Z. Zhu, H.-Y. Zhou and N. Bin, Non-Cooperative Stochastic Differential Game Theory of Generalized Markov Jump Linear Systems. Switzerland: Springer, 2017.

[7] I. R. Petersen and D. C. McFarlane, "Optimal guaranteed cost control and filtering for uncertain linear systems," IEEE Transactions on Automatic Control, vol. 39, no. 9, pp 1971-1977, 1994.

[8] V. Dragan, T. Morozan and A. M. Stoica, Mathematical Methods in Robust Control of Linear Stochastic Systems. New York: Springer, 2006. 
[9] X. Li and and C. E. D. Souza, "Criteria for robust stability and stabilization of uncertain linear systems with state delay," Autornatica, vol. 33, no. 9, pp 1657-1662, 1997.

[10] H. Mukaidani, "Robust guaranteed cost control for uncertain stochastic systems with multiple decision makers," Automatica, vol. 45, no. 7, pp. 1758-1764, 2009.

[11] S. Boyd and L. Vandenberghe, Convex Optimization. Cambridge: Cambridge University Press, 2004.

[12] J. Engwerda, LQ Dynamic Optimization and Differential Game. Chichester: John Wiley and Sons Inc., 2005. 